\title{
Oncological consequence of emergent resection of perforated colon cancer with complete obstruction after stent insertion as a bridge to surgery
}

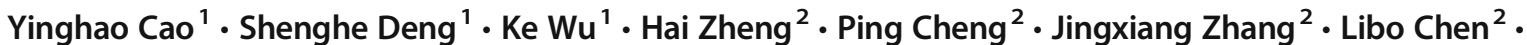 \\ Shouyuan Tang ${ }^{3} \cdot$ Pingfa Wang ${ }^{4}$ - Xiaofeng Liao ${ }^{5}$. Yongkang Zhang ${ }^{5} \cdot$ Gaobo Zhu $^{6} \cdot$ Qiaoyun Tong ${ }^{7}$. Jiliang Wang ${ }^{1}$. \\ Jinbo Gao ${ }^{1}$. Xiaoming Shuai ${ }^{1} \cdot$ KaiXiong Tao $^{1} \cdot$ Guobin Wang ${ }^{1} \cdot$ Jiang $\mathrm{Li}^{1} \cdot \mathrm{Kailin} \mathrm{Cai}^{1}$
}

Accepted: 25 July 2018 / Published online: 8 August 2018

(C) The Author(s) 2018

\begin{abstract}
Purpose Colonic perforation is a life-threatening complication after colonic stent insertion as a bridge to surgery for acute obstruction caused by colorectal cancer. The oncological consequence of colonic perforation after emergent surgical intervention was unknown. The aim of this short communication was to investigate whether or not the perforation and emergent surgery had obviously impact on the peritoneal recurrence and long-term survival of patients.

Methods Data of the patients who underwent colorectal stenting as a bridge to surgery in 5 years from 2012 to 2017 was collected by the Endoscopical Surgery Group of Hubei. The perforated cases treated by emergent operation were retrospectively analyzed. Results During 5 years from 2012 to 2017, 116 cases of colorectal stenting as a bridge to surgery had been performed, and 7 patients had perforation after stent placement and treated by emergent surgery, including 1 case of synchronic liver metastasis treated by one-stage metastasectomy. One of the 7 patients died of septic shock after operation, and the remaining patients were followed up for 6-60 months. There was no evidence of abdominal implantation or extra-abdominal metastasis.

Conclusion This small case series implicated that colonic perforation after stent insertion for malignant colorectal obstruction treated by emergent surgery might not obviously increase the peritoneal implantation and metastasis.
\end{abstract}

Keywords Self-expanding metal stent $\cdot$ Bridge to surgery $\cdot$ Colon cancer $\cdot$ Intestinal perforation $\cdot$ Metastasis

\section{Introduction}

Colorectal cancer ranks the third most commonly malignant neoplasm in males and the second in females, with an estimated 1.4 million cases and 693,900 deaths occurring in 2012 [1]. About 7 29\% of all colorectal cancer patients would experience partial or complete bowel obstruction, and the classical

Kailin Cai

caikailin@hust.edu.cn

1 Department of Gastrointestinal Surgery, Union Hospital, Tongji Medical College, Huazhong University of Science and Technology, Wuhan 430022, China

2 Department of Emergency and Trauma Surgery, Union Hospital, Tongji Medical College, Huazhong University of Science and Technology, Wuhan 430022, China

3 Department of General Surgery, Huangshi Central Hospital, Huangshi 435000, Hubei Province, China intervention for acute intestinal obstruction was surgery. Colon stent was first introduced in the early 1990s as a tool to treat malignant colonic obstruction [2]. Thereafter, the placement of a self-expanded metal stent (SEMS) had been effectively used in cases of acute malignant obstruction as a bridge to surgery. In acute obstruction relieved by the stenting, the patient would have more chance of primary anastomosis

4 Department of Digestive Disease, Huangshi Central Hospital, Huangshi 435000, Hubei Province, China

5 Department of General Surgery, Affiliated Hospital of Hubei University of Arts and Science, Xiangyang 441021, Hubei Province, China

6 Department of Emergency and Trauma Surgery, Yichang Central People's Hospital, China Three Gorges University, Yichang 443003, Hubei Province, China

7 Department of Digestive Disease, Yichang Central People's Hospital, China Three Gorges University, Yichang 443003, Hubei Province, China 
without ostomy, lower perioperative mortality, and lower overall complication rate.

But the stenting at the same time might cause some complications. The most common complications were perforation, stent displacement, and re-obstruction, of which perforation was the most concerned, because it was life-threatening. Perforation was reported in $0-16 \%$ patients with colorectal SEMS placement [3]. When fecal leakage and peritonitis happened, emergent surgical intervention should be applied as soon as possible.

The emergent surgery was usually effective to rescue the life. Nevertheless, there was yet question. Whether the colonic perforation after stent insertion increased the risk the patient's the long-term outcome due to potential peritoneal seeding of cancer cells? Here, we reported the oncological results of seven cases of perforation after SEMS.

\section{Cases}

Seven cases of perforation were documented in our study group, three males and four females, with an age between 53 and 79 years old, all with a medical history of acute complete obstruction in the colon. Thin-layer CT scan of the whole abdomen was done for each case soon after the patient's visit. Single stricture in any part of the colon was set as the inclusion criteria of stenting procedure. One patient had resectable hepatic metastases. The stents had been inserted through the stricture and released successfully in all but one patient. In this patient, the stent was partially distracted outside the colon into the mesocolon at an angle of the stricture located in transverse colon, but small amount of feces was noticed flowing out through the stent during the stenting procedure. The obstruction symptoms did not alleviate after $24 \mathrm{~h}$ and then the perforation and stent displacement was noticed after a CT scan. As for the other patients, the median time interval to perforation was 2 days (range $0-4$ days). Three patients underwent laparoscopic surgery and the other four patients underwent laparotomy, two patients underwent primary radical resection and anastomosis of tumor, and the other five patients underwent primary radical resection with ileostomy. One patient with ASA III before the stent procedure died of a variety of companied diseases after the emergent ileostomy in one hospital of our study group with low volume of stent procedure. In all seven, four were poorly differentiated, two moderately differentiated, and one moderately poorly differentiated adenocarcinoma. The remaining six patients attend scheduled follow-up visits, and the longest follow-up time is more than 5 years. No patient died during the follow-up period. The patient who had resectable liver metastases was treated with simultaneous colectomy and lobectomy followed by six cycles of chemotherapy. None of the six patients developed cancer recurrence.

\section{Discussion}

Patients with acute intestinal obstruction usually had various accompanied conditions such as dehydration, anemia, metabolic disorders, malnutrition, and acid-base imbalance. Compared with elective primary resection, emergency surgery for acute colonic obstruction carries high morbidity and mortality. Nevertheless, in an emergent surgery, the quality of the operation might hardly meet the standard of elective surgery. It has been reported that the total number of lymph nodes retrieved was significantly less than that of the elective operation. After an emergent colectomy, either the rate of protective ileostomy or the permanent colostomy was higher than that after elective surgery. The rate of overall complication after emergent surgery was also shown to be higher by several meta-analyses [4]. These problems greatly impacted the quality of life and cost of living of colon cancer patients.

Many studies have proved that SEMS was safe and effective to relieve the obstruction either in a bridge to surgery policy or in palliative treatment for malignant colon obstruction. But its complications should also be taken into consideration when we make choices. The most serious complication was colonic perforation, and it could cause severe abdominal infection, aggravate the condition, and even lead to death, if the condition had not been soon controlled.

We had started the bridge to surgery for malignant colorectal obstruction since 2008 and established the Endoscopical Surgery Group to collaborate on the research in 2012. At present, there were endoscopic surgery teams in four tertiary hospitals to carry out stent placement followed by operations, with 116 cases in total. We performed emergency operation on seven patients with perforation and followed up after the operation.

The time of perforations was reported mainly occurred within 30 days after stent placement in bridge to surgery, and almost half of them were noted acutely or sub-acutely (during or within 1 day of the procedure). In our cases, the perforation occurred to a patient in 3 days after the procedure, and the other six patients occurred within $24 \mathrm{~h}$ after the procedure.

The reasons for the perforation after stent implacement were categorized into the stent-related and the non-stent-related factors. Stent-related perforation was caused by mechanical properties of the stent. Non-stent-related factors included the use of guide wires, pre-dilation, and balloon expansion. In addition, anatomic factors, chemotherapeutic agents, steroids, and radiotherapy or laser therapy had also been identified as risk factors for perforation. We analyzed the perforation in our series after the operation. In three cases, the perforated site located at the edge of the stent might be caused by the repeated friction of the end of the stent, and in four cases, the tumor perforated; therefore, it might be caused by the stent expansion because we never used balloon expansion during the procedure. In one case, the stent went inserted through most part of the lumen and then passed out at an angle of the long tumor, into the mesocolon without peritonitis. 
Kim et al. [5] have addressed the possibility of adverse impact on long-term survival in patients treated with self-expanding metallic stent insertion as a bridge to surgery compared to nonobstructing elective surgery. However, it was unclear if it was mainly related to the underlying obstruction or to SEMS [5]. Some studies have reported that SEMS placement may be associated with a higher tumor stage. The expansion of malignant obstruction with stents might promote the migration of tumor cells and thus, might increase the risk of systemic metastasis [6]. A retrospective study reported that the incidence of perineural infiltration after colonic stent implantation was significantly higher (76 to $51.4 \%$; $P=0.033$ ) [7]. The potential of tumor dissemination during the stent insertion procedure had not been well studied. Nevertheless, inadvertent intraoperative perforation during curative resection had also been reported to lead to spillage of cancer cells and adversely affected the long-term survival [8].

The peritoneal recurrence in patients with malignant colorectal obstruction treated with SEMS was interesting. Su Jin Kim et al. [9] reported that perforation may result in the intraabdominal tumor seeding and tumor growth. Their results support the theoretical association between perforation and intraperitoneal tumor seeding. The long-term oncological outcomes after emergency operation of colonic perforation due to stent insertion were followed. In our series of six cases that survived the surgery, no peritoneal implantation and recurrence was verified. The result was implicative although only four cases had been followed up for more than 2 years. The recurrence of colorectal cancer was most significantly happen in 2 years after the curative surgery [10], and Park [11] reported that nearly $95 \%$ of recurrences occurred within 3 years after surgery.

Our follow-up results showed that perforation after stenting did not significantly increase the risk of tumor growth and recurrence in 2 years of follow-up. The discrepancy with other report which showed elevated intraperitoneal recurrence might be explicated by an early diagnosis and also early treatment of the perforation. The result supported a further study on the stent bridge to surgery [12].

\section{Conclusion}

Colonic perforation after stent insertion as a bridge to surgery for malignant colorectal obstruction might not increase significantly the risk of peritoneal implantation.

\footnotetext{
Author contributions Yinghao Cao, Shenghe Deng, Jiang Li, Ke Wu, Hai Zheng, Ping Cheng, Jingxiang Zhang, Libo Chen, Shouyuan Tang, Pingfa Wang, Xiaofeng Liao, Yongkang Zhang, Gaobo Zhu, Qiaoyun Tong, Jiliang Wang, Jinbo Gao, Xiaoming Shuai, KaiXiong Tao, Guobin Wang, and Kailin Cai all met all the criteria for the definition of authorship and contributed substantially to the manuscript. Yinghao Cao, Shenghe Deng, and Ke Wu are the first authors, and they contributed equally to this work. Jiang $\mathrm{Li}$ and Kailin Cai are the corresponding authors.
}

Funding information This study was supported by grants from the Science and Technology Department of Hubei Province (No. 2018CFC884), the Wu Jieping Medical Foundation of China (No. 320.2710.1843), and Clinical Research Physician Program of Tongji Medical College, HUST.

\section{Compliance with ethical standards}

Conflict of interest The authors declare that they have no conflict of interest.

Open Access This article is distributed under the terms of the Creative Commons Attribution 4.0 International License (http:// creativecommons.org/licenses/by/4.0/), which permits unrestricted use, distribution, and reproduction in any medium, provided you give appropriate credit to the original author(s) and the source, provide a link to the Creative Commons license, and indicate if changes were made.

\section{References}

1. Torre LA, Bray F, Siegel RL, Ferlay J, Lortet-Tieulent J, Jemal A (2015) Global cancer statistics, 2012. CA Cancer J Clin 65:87-108

2. Sebastian S, Johnston S, Geoghegan T, Torreggiani W, Buckley M (2004) Pooled analysis of the efficacy and safety of self-expanding metal stenting in malignant colorectal obstruction. Am J Gastroenterol 99:2051-2057

3. Nitta T, Kataoka J, Ohta M, Fujii K, Tominaga T, Inoue Y, Kawasaki H, Ishibashi T (2017) Clinical outcomes of selfexpandable metal stent (SEMS) placement as palliative treatment for malignant colorectal obstruction: a single-center study from Japan. Ann Med Surg (Lond) 19:33-36

4. Zhao X, Liu B, Zhao E et al (2014) The safety and efficiency of surgery with colonic stents in left-sided malignant colonic obstruction: a meta-analysis. Gastroenterol Res Pract 2014:407325

5. Kim JS, Hur H, Min BS, Sohn SK, Cho CH, Kim NK (2009) Oncologic outcomes of self-expanding metallic stent insertion as a bridge to surgery in the management of left-sided colon cancer obstruction: comparison with nonobstructing elective surgery. World J Surg 33:1281-1286

6. Thorlacius H (2008) Tumour cell dissemination following endoscopic stent insertion. Br J Surg 94:1151-1154

7. Kim HJ, Choi GS, Park JS, Park SY, Jun SH (2013) Higher rate of perineural invasion in stent-laparoscopic approach in comparison to emergent open resection for obstructing left-sided colon cancer. Int J Clolrectal Dis 28:407-414

8. Slanetz CJ (1984) The effect of inadvertent intraoperative perforation on survival and recurrence in colorectal cancer. Dis Colonrectum 27:792-797

9. Kim SJ, Kim HW, Park SB, Kang DH, Choi CW, Song BJ, Hong JB, Kim DJ, Park BS, Son GM (2015) Colonic perforation either during or after stent insertion as a bridge to surgery for malignant colorectal obstruction increases the risk of peritoneal seeding. Surg Endosc 29:3499-3506

10. Park YJ, Park KJ, Park JG, Lee KU, Choe KJ, Kim JP (1999) Prognostic factors in 2230 Korean colorectal cancer patients: analysis of consecutively operated cases. World J Surg 23:721-726

11. Saha S, Nora D, Wong JH, Weise D (2000) Sentinel lymph node mapping in colorectal cancer-a review. Surg Clin North Am 80: 1811-1819

12. Manfredi S, Sabbagh C, Vanbiervliet G, Lecomte T, Laugier R, Karoui M (2014) Place of colorectal stents in therapeutic management of malignant large bowel obstructions. Endoscopy 46:546552 